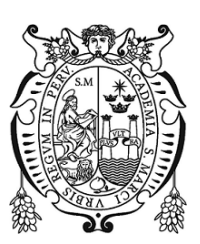

Revista de Investigación de Física 24(1), (Ene-Jun 2021)

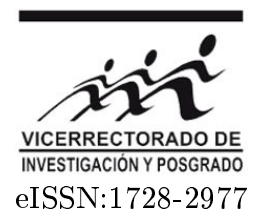

\title{
Identificación de arcillas de óxidos de hierro en las plantaciones de caña de azúcar en el distrito de Pomalca
}

\author{
María Cerón ${ }^{* 1}$, Franko Urcia ${ }^{2}$, Jenny Montoya ${ }^{3}$, Alejandro Trujillo y Jorge Bravo ${ }^{1}$ \\ ${ }^{1}$ Universidad Nacional Mayor de San Marcos. Facultad de Ciencias Físicas. Laboratorio de Análisis de \\ Suelos. Lima, Perú \\ ${ }^{2}$ Universidad Nacional Pedro Ruiz Gallo, Lambayeque, Perú \\ ${ }^{3}$ Universidad César Vallejo. Trujillo, Perú
}

Recibido 05 julio 2020 - Aceptado 22 diciembre 2020

\begin{abstract}
Resumen
Hemos identificado la presencia de óxidos de hierro, arcillas y otros minerales presentes en la fracción arcillosa de seis muestras provenientes de tres calicatas obtenidas de los suelos de las plantaciones de caña de azúcar de la empresa agroindustrial Pomalca S.A.A. Estas plantaciones están localizadas en el Distrito de Pomalca, Provincia de Chiclayo, Región Lambayeque, Perú. Las calicatas fueron identificadas como Estrella, Estreno y Mochica. La difracción de rayos X y espectroscopía Mössbauer de transmisión, complementados con los análisis físico-químicos de rutina, permitieron obtener resultados que resolvieron la identificación de las fases estructurales y mineralógicas de óxidos de Fe presentes adjudicados a la presencia de hematita, así como sitios de $\mathrm{Fe}^{2+}$ localizados en la estructura de los minerales arcillosos de illita y/o montmorillonita, y un sitio de $\mathrm{Fe}^{3+}$ localizado en la estructura de la caolinita.
\end{abstract}

Palabras clave: Espectroscopia Mössbauer de transmisión, difractrometría de rayos X, arcillas, minerales arcillosos, suelos..

\section{Identification of clays and iron oxides in the sugar cane plantations of Pomalca distric's}

\begin{abstract}
We have identified the presence of Fe oxides, clays and other minerals in the clay fractions of six samples gathered at three pits practiced in soils of sugar cane plantations of the agroindustrial company Pomalca S.A.A. These plantations are located in the District of Pomalca, Province of Chiclayo, Lambayeque Region, Peru. The pits were identified as: Estrella, Estreno and Mochica. The X-ray diffractometry and transmission Mössbauer spectroscopy techniques, complemented with routine physical-chemical analyses, allowed us to obtain results that resolved the identification of the structural and mineralogical phases of the iron oxides present which were assigned to hematite, as well as $\mathrm{Fe}^{2+}$ sites located in the structure of clays minerals such as Illite and/or montmorillonite, and a $\mathrm{Fe}^{3+}$ site assigned to the structure of kaolinite.
\end{abstract}

Keywords: transmission Mössbauer spectroscopy and X-ray diffractometry, clays, clay minerals, soils..

\section{Introducción}

Numerosas investigaciones sobre la presencia de los óxidos de hierro en suelos se realizan en diferentes instituciones de Latinoamérica y el mundo con diferentes objetivos basados en la diversidad edafológica que presentan [Cornell y Schwertmann, 2003]. En la actualidad, en México, los óxidos de hierro se encuentran en todos los suelos en cantidades diversas y son utilizados como indicadores de procesos pedogenéticos [Hidalgo et al., 2010], en Indonesia, estudian el contenido de la distribución de los óxidos de $\mathrm{Fe}, \mathrm{Al}$ y $\mathrm{Si}$ en tierras secas [Sufardi, et al., 2019], mientras que en Chile, se estudia la mineralogía de los óxidos de hierro de suelos derivados de cenizas volcá- 
nicos [Pizarro, et al., 2017]. De esta forma, en la misma línea de investigación, se continúan con los trabajos desarrollados en el Laboratorio de Análisis de Suelos de la Facultad de Ciencias Físicas de la Universidad Nacional Mayor de San Marcos, (LAS-FCF; UNMSM), sobre la identificación de minerales arcillosos, de óxidos, sesquióxidos e hidróxidos de hierro, así como el revestimiento estructurado en las diferentes muestras de suelos agrícolas extraídas de las diferentes regiones del Perú [Cerón, 2001; Trujillo, 2002; Mejía, 2013; Nima, 2008; Tanejaa y Rajb, 1993; Stevens, 1998; Richards, 1985; Perugachi et al., 2006; Patel, 2014; Nigam et al., 2004; Flores y Alcala, 2010] y ahora presentamos resultados del trabajo preliminar, que tiene por objetivo identificar óxidos de hierro y arcillas presentes en las fracciones arcillosas de los suelos de las plantaciones de caña de azúcar.

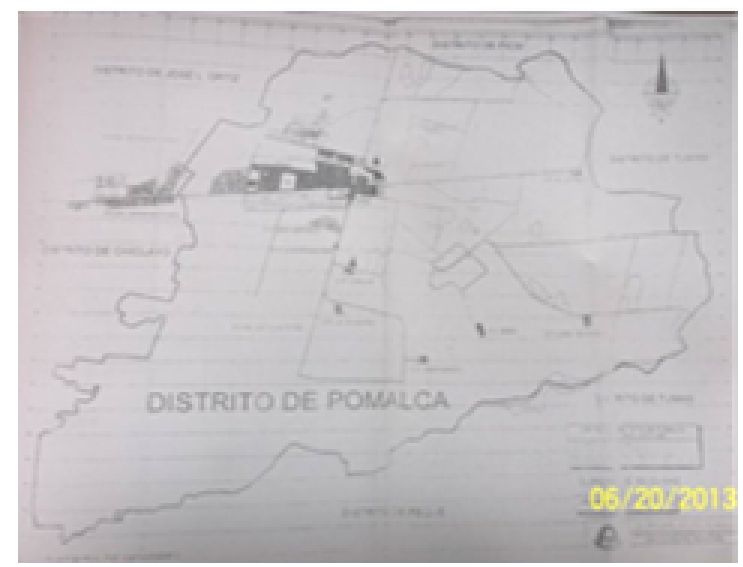

Figura 1: Ubicación del Distrito de Pomalca, Departamento de Lambayeque.

Estas plantaciones están ubicadas en el Distrito de Pomalca, Provincia de Chiclayo, Región Lambayeque, en la Costa Norte del Perú, a $770 \mathrm{~km}$ al norte de la ciudad de Lima y a $7 \mathrm{~km}$ de la ciudad de Chiclayo, Región Lambayeque [https://www.pomalca.com.pe/pomalcaweb/em_ quienessomos.php]; aproximadamente entre las coordenadas geográficas $6^{\circ} 44^{\prime} 01^{\prime \prime}$ y $6^{\circ} 49^{\prime} 01^{\prime \prime}$ de latitud sur, y entre $79^{\circ} 42^{\prime} 59^{\prime \prime}$ y $79^{\circ} 48^{\prime} 09^{\prime \prime}$ de longitud oeste del meridiano de Greenwich y a 40 m.s.n.m. (Ver figura 1). El clima es cálido-templado, regulado por la Cadena Occidental de los Andes, la corriente marina de Humboldt y el fenómeno de "El Niño". La temperatura fluctúa entre los $31.6^{\circ} \mathrm{C}$ en verano y $15^{\circ} \mathrm{C}$ en invierno; la humedad relativa varía entre el $55 \%$ y $60 \%$; las precipitaciones pluviales son de $77 \mathrm{~mL}$ anuales. Ostentan una fisiografía típica de los valles de la Costa Norte, presentando las siguientes clases de suelo: suelos arenosos, areno-arcillosos, francos, arcillosos y arcillosos-limosos; los cuales se dedican al cultivo de la caña de azúcar. La extracción de las muestras se realizó in situ, en coordinación y colaboración con el personal de la Empresa, se procedió a realizar una apertura de tres calicatas por campo, y se tomaron dos muestras por calicata, a superficie y a profundidad: 0-0.50 m y de $0.50-1.00 \mathrm{~m}$, para las muestras denominadas como Estrella; de $0-0.35 \mathrm{~m}$ y de $0.35-0.70 \mathrm{~m}$ para las rotuladas como Estreno, y de $0-0.40 \mathrm{~m}$ y de $0.40-0.80 \mathrm{~m}$ para la muestra denominada Mochica.

Por tanto, se obtuvieron seis muestras en total provenientes de las tres calicatas. Luego fueron llevadas al Laboratorio de Análisis de Suelos de la Facultad de Ciencias Físicas (FCF) de la UNMSM, para sus análisis posteriores. La Empresa Pomalca S.A.A. manifestó su preocupación por pérdidas de niveles tanto de producción (disminución de superficies de cultivo) como de productividad (disminución de los rendimientos), siendo necesario afrontar estos dos factores con políticas y estrategias de inversión en el corto, mediano y largo plazo. Para la reversión de su situación, implica efectuar grandes inversiones en proyectos de rehabilitación y mejoramiento de suelos, llevar a cabo un estudio edafológico detallado, que permita identificar y planificar el programa de inversiones más adecuado, tendiente a cubrir las 11,265 ha con que cuenta la institución.

Desde el punto de vista de la agronomía, es de vital importancia conocer la composición mineralógica de los suelos para llevar a cabo las actividades de planificación sobre el uso y recuperación de suelos [Thompson y Troeh, 2002]. Este trabajo reporta resultados preliminares, basados en el estudio y la caracterización mineralógica de los suelos de plantaciones de caña de azúcar con la finalidad de determinar su clasificación textural, los óxidos de hierro, tipos de arcilla y otros minerales presentes en su composición.

\section{Materiales y métodos}

Para el análisis instrumental de las muestras se procedió de la siguiente manera:

\section{Preparación de las muestras}

Para todo los procedimientos, los ensayos y análisis de las muestras desde su extracción, se siguieron los protocolos del Manual del Laboratorio de análisis de suelos [Bravo et al., 2000]. No existe una estandarización de todos los métodos de caracterización física, sin embargo, este manual empleado se ajusta a toda la metodología que presenta la Sociedad Americana de la Ciencia del Suelo [Dane and Topp, 2002] y se fundamenta con los análisis por difracción de rayos X [Moore y Reynolds, 1997].

Para este trabajo, se extrajeron in situ 6 muestras de suelos, las cuales fueron rotuladas como Estrella, Estreno y Mochica. Se procedió a la apertura de una calicata por campo, obteniéndose de cada calicata dos muestras, cada una a una profundidad determinada: $0-0.50 \mathrm{~m}$ y de 


\begin{tabular}{|l|c|c|c|}
\hline \multirow{2}{*}{ Muestras/profundidad } & Humendad & $\mathrm{pH}$ & C.E. \\
\cline { 2 - 4 } & $\%$ & $1: 1$ & $\mathrm{dS} / \mathrm{m}$ \\
\hline ESTRENO $(0.00-0.35 \mathrm{~m})$ & 1.276 & 9.0 (fuertemente alcalina) & 7.7 (salino) \\
\hline ESTRENO $(0.35-0.70 \mathrm{~m})$ & 1.286 & 8.9 (fuertemente alcalina) & 5.5 (salino) \\
\hline ESTRELLA $(0.00-0.50 \mathrm{~m})$ & 1.174 & 8.5 (fuertemente alcalina) & 18.9 (extremadamente salino) \\
\hline ESTRELLA $(0.50-1.00 \mathrm{~m})$ & 1.125 & 9.5 (fuertemente alcalina) & 12.4 (fuertemente salino) \\
\hline MOCHICA $(0.00-0.40 \mathrm{~m})$ & 1.386 & 8.3 (alcalina) & 2.8 (ligeramente salino) \\
\hline MOCHICA $(0.40-0.80 \mathrm{~m})$ & 1.164 & 8.4 (alcalina) & 6.9 (salino) \\
\hline
\end{tabular}

Tabla 1: Análisis físico de las muestras.

0.50 - $1.00 \mathrm{~m}$ para Estrella; $0-0.35 \mathrm{~m}$ y $0.35-0.70 \mathrm{~m}$ para Estreno y de $0-0.40 \mathrm{~m}$ y de $0.40-0.80 \mathrm{~m}$ para Mochica. Las muestras fueron recolectadas y puestas en custodia en el Laboratorio de Análisis de Suelos (LAS) de la Facultad de Ciencias Físicas de la Universidad Nacional Mayor de San Marcos (UNMSM). Se procedió a secarlas en una mufla a $27^{\circ} \mathrm{C}$, molerlas en un mortero de ágata, luego tamizarlas en mallas de $2 \mathrm{~mm}, 90 \mu \mathrm{m}, 80 \mu$ m y $38 \mu \mathrm{m}$, para someterlas luego a los análisis físicoquímicos de rutina. Se obtuvo de cada una de ellas la fracción arcillosa por el método de sedimentación [Bravo et al., 2000] éste proceso es una técnica de laboratorio, cuyos principios bajo el cual se fundamentan estos cálculos están basados en la Ley de Stokes, orientada a separar las fracciones granulométricas, en el que la velocidad de caída de una partícula en un fluido depende, entre otros factores, de su tamaño; así aquellas más grandes caerán más rápidamente que las de diámetro inferior, por tanto; consiste en calcular empíricamente la velocidad de caída de las partículas en un fluido. Finalmente, las fracciones obtenidas, serán analizadas por las técnicas analíticas de difractometría de rayos-X (DRx) y espectroscopia Mössbauer de transmisión (EMT). Se empleó $250 \mathrm{mg}$ para los análisis por EMT usando un porta muestra de aproximadamente de $0.954 \mathrm{~cm}^{2}$ de área.

\section{Análisis físico-químico}

Para las muestras seleccionadas se determinó el contenido de agua ( \% agua), el pH, sales solubles (conductividad eléctrica $\mathrm{CE}$ ) empleando un medidor de electrodo modelo pH Meter 110, marca OAKTON, estos resultados de los análisis físico-químicos se observan en la tabla 1. La determinación de la clase textural (método del hidrómetro), nos permite determinar el porcentaje de arena, limo y arcilla que contiene una muestra de suelo, para ello se sigue un procedimiento de rutina [Bravo et al., 2000] y se determina utilizando el "triángulo textural", de acuerdo a las siguientes relaciones:

$$
\begin{array}{r}
\% \text { Arena }=100-(\text { lect.correg. } 40 \mathrm{~s} \times 100 / \text { peso.muestra }) \\
\% \text { Arcilla }=\text { lect.correg. } 2 \text { hr } \times 100 / \text { peso.muestra } \\
\% \text { Limo }=100-(\% \text { arena }+\% \text { arcilla })
\end{array}
$$

La lectura corregida depende de la temperatura que posea la mezcla para ello se precisa hallar el factor de corrección (F. C.), esta se determina de acuerdo a la siguiente relación:

$$
\text { F.C. }=7.2+0.36(\text { Temp })
$$
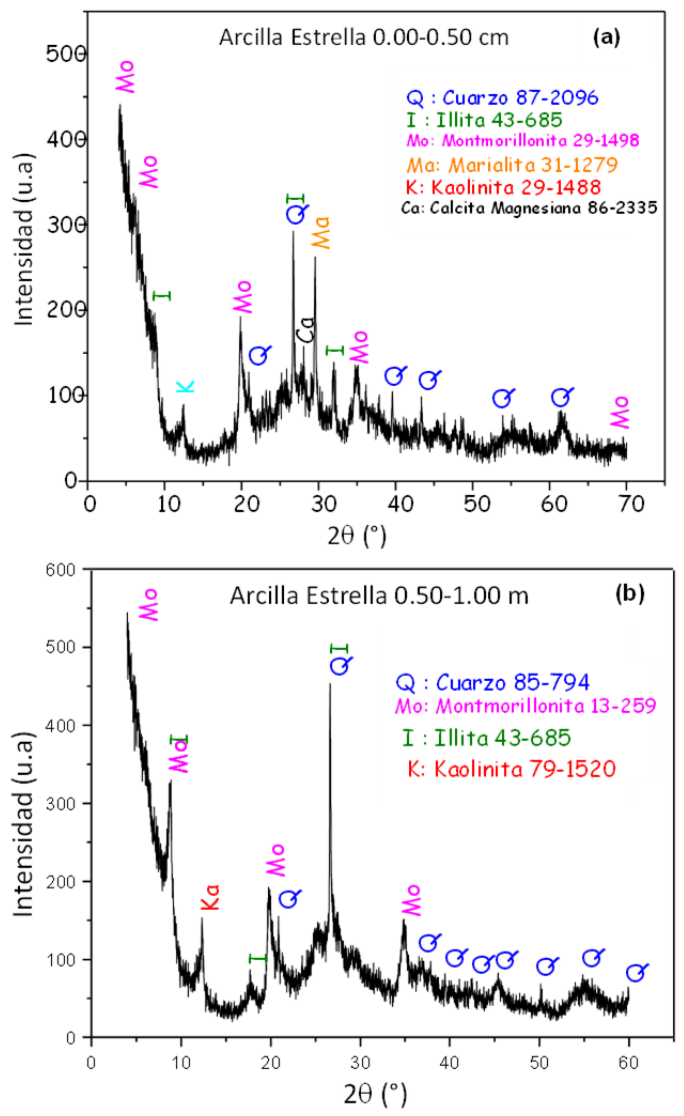

Figura 2: Patrones de difracción de rayos $\mathrm{X}$ de la muestra arcilla Estrella a una profundidad de: (a) $0.00-0.50 \mathrm{~m}$, mostrando reflexiones de las fases del cuarzo (Q), illita (I), montmorillonita (Mo), marialita (Ma), caolinita (K) y calcita magnesiana (Ca) y (b) $0.50-1.00 \mathrm{~m}$., demostrando reflexiones de las fases del cuarzo (Q), illita (I), montmorillonita (Mo) y caolinita $(\mathrm{K})$. 


\begin{tabular}{|l|c|c|c|c|}
\hline \multirow{2}{*}{ Muestras/profundidad } & \multicolumn{4}{|c|}{ Análisis textural } \\
\cline { 2 - 5 } & \% Arena & \% Arcilla & \% Limo & Textura \\
\hline ESTRENO $(0.00-0.35 \mathrm{~m})$ & 66 & 14 & 20 & Fo.Ao \\
\hline ESTRENO $(0.35-0.70 \mathrm{~m})$ & 68 & 15 & 17 & Fo.Ao \\
\hline ESTRELLA $(0.00-0.50 \mathrm{~m})$ & 64 & 12 & 24 & Fo.Ao \\
\hline ESTRELLA $(0.50-1.00 \mathrm{~m})$ & 75 & 14 & 11 & Fo.Ao \\
\hline MOCHICA $(0.00-0.40 \mathrm{~m})$ & 66 & 13 & 21 & Fo.Ao \\
\hline MOCHICA $(0.40-0.80 \mathrm{~m})$ & 72 & 20 & 8 & Fo.Ar.Ao \\
\hline
\end{tabular}

Tabla 2: Análisis textural de las muestras (Fo.Ao = franco arenoso; Fo.Ar.Ao = franco arcilloso arenoso ).

Luego de hallar el factor de corrección, sumar ó restar éste a las lecturas medidas. La relación para determinar el factor de corrección esta dado para un hidrómetro a $68^{\circ} \mathrm{F}$. Es recomendable, remover la materia orgánica, sales ó carbonatos, porque la presencia de ellas disminuye la precisión de los resultados para estudios edafológicos. Los principios bajo el cual se fundamentan estos cálculos están basados en la Ley de Stokes. En la tabla 2 se observan resultados de los análisis de textura de las muestras de suelos, se empleó como referencia el "Manual de Laboratorio de Análisis de Suelos" [Bravo, et al., 2000] del LAS-FCF; UNMSM.

\section{Técnicas físicas aplicadas}

\section{Medidas y análisis por difractometría de ra- yos X (DRx)}

El equipo de difracción de rayos $\mathrm{x}$, que nos permite la obtención de los datos, corresponde a un difractómetro marca Brucker, donde empleamos una radiación de $\mathrm{Cu}-\mathrm{K} \alpha(\lambda=1.54178 \AA)$, con un goniómetro vertical y con monocromador. El barrido angular fue de $4^{\circ}<2 \theta<70^{\circ}$, a superficie (profundidad de $0.00-0.50 \mathrm{~m}$ ) sólo para la muestra Estrella, y para la profundidad de $0.50-1.00 \mathrm{~m}$, así como para la muestra denominada Estreno y Mochica extraídas a dos profundidades, se registraron con un ángulo de barrido de $4^{\circ}<2 \theta<60^{\circ}$. Se utilizó un avance de $0.02^{\circ} /$ paso de 2 segundos para todas las muestras.

Posterior a la recolección de datos, procedemos a la identificación estructural cualitativa de los compuestos presentes en las muestras, empleando una base de datos Crystallographica-Search Match (CSM). CSM Search Macht 1.1, Team LND. Versión 2, que es un programa de búsqueda con las bases de datos en PDF del Centro Internacional de Datos de Difracción (CIDD).

\section{Medida y análisis por espectroscopia Möss- bauer de transmisión (EMT)}

Para obtener información más detallada sobre los compuestos que contienen Fe se recurrió a la técnica de EMT. Las mediciones de las muestras se realizaron a tem- peratura ambiente (TA) en el Laboratorio de Arqueometría del Instituto de Investigación de la Facultad de Ciencias Físicas (UNMSM). Los espectros Mössbauer fueron tomados en geometría de transmisión usando una fuente radiactiva de ${ }^{57} \mathrm{Co}$ en una matriz de $\mathrm{Rh}$ con una actividad aproximada de $5 \mathrm{mCi}$. Los espectros fueron analizados utilizando el programa Normos de Brand en la versión de sitios cristalinos [Brand, 1995]; se empleó aprox. 250 mg de muestra en polvo para obtener un adecuado grosor óptico.

\section{Discusión y resultados}

Los resultados de los análisis físico-químicos se observan en la Tabla 1: incluyen el porcentaje de humedad, el grado de acidez $(\mathrm{pH})$ y la conductividad eléctrica (CE). La CE es un parámetro que muestra la capacidad de una disolución para transmitir corriente eléctrica, este valor de la CE depende de la concentración de sales disueltas. La Tabla 2 presenta los resultados del análisis textural, donde se observa una variedad de porcentajes de fracción arcilla de no más del $45 \%$.

Los resultados obtenidos por DRx se observan en los difractogramas de las Figuras 2, 3 y 4; en todos los difractogramas observamos picos anchos; es probable que se trate de una muestra con poca cristalinidad y/o amorfa, que puede contener abundante materia orgánica que no permite una mejor resolución para la identificación de los picos patrones de los minerales. Asimismo en todas las muestras observamos al mineral primario principal, el cuarzo $\left(\mathrm{SiO}_{2}\right)$, y fases estructurales de la illita $\left(\mathrm{K}, \mathrm{H}_{3} \mathrm{O}\right)(\mathrm{Al}, \mathrm{Mg}, \mathrm{Fe})_{2}(\mathrm{Si}, \mathrm{Al})_{4} \mathrm{O}_{10}\left[(\mathrm{OH})_{2},\left(\mathrm{H}_{2} \mathrm{O}\right)\right]$ y montmorillonita [(Na, Ca $) 0,3(\mathrm{Al}, \mathrm{Mg}) 2 \mathrm{Si} 4 \mathrm{O} 10(\mathrm{OH})_{2}$ $\mathrm{n}\left(\mathrm{H}_{2} \mathrm{O}\right)$ ] (http://webmineral.com/data/Illite. shtml\#.XpoivUYzbIU). En los difractogramas de la Figura 2(a), correspondiente a la muestra Estrella superficial $(0.00-0.50 \mathrm{~m})$, a diferencia de la Figura 2(b) que corresponde a la muestra arcilla Estrella de mayor profundidad (0.50-1.00 m), encontramos que, a mayor profundidad, dos fases minerales: marialita y calcita magnesiana, desaparecen; esto se observa en el rango $26.4^{\circ}<2 \theta<60^{\circ}$, que probablemente sea el resultado del lavado por la erosión de los suelos, las precipitaciones fluviales y/o 


\begin{tabular}{|l|c|c|c|c|c|}
\hline Muestras & $\begin{array}{c}\text { Estrella } \\
(0.00-0.50 \mathrm{~m})\end{array}$ & $\begin{array}{c}\text { Estrella } \\
(0.50-1.00 \mathrm{~m})\end{array}$ & $\begin{array}{c}\text { Estreno } \\
(0.00-0.35 \mathrm{~m})\end{array}$ & $\begin{array}{c}\text { Estreno } \\
(0.35-0.70 \mathrm{~m})\end{array}$ & $\begin{array}{c}\text { Mochica } \\
(0.40-0.80 \mathrm{~m})\end{array}$ \\
\hline $\begin{array}{l}\text { Fases } \\
\text { estructurales }\end{array}$ & Código & Código & Código & Código & Código \\
\hline Cuarzo & $87-2096$ & $85-794$ & $75-443$ & $78-2915$ & $87-2096$ \\
\hline Illita & $43-685$ & $43-685$ & $9-343$ & $9-343$ & $26-911$ \\
\hline Montmorillonita & $29-149$ & $13-259$ & $13-259$ & $13-259$ & $13-135$ \\
\hline Marialita & $31-1279$ & & $2-412$ & $29-1496$ & $89-3568$ \\
\hline Kaolinita & $29-1488$ & $79-1520$ & $72-2300$ & $72-2300$ & \\
\hline $\begin{array}{l}\text { Calcita } \\
\text { magnesiana }\end{array}$ & $86-2335$ & & & & \\
\hline
\end{tabular}

Tabla 3: Códigos de las fases estructurales de cada muestra.

fertilizantes empleados, los cuales deben haber revestido la arcilla y los minerales arcillosos de la parte superficial del suelo.

En los difractogramas de la Figura 3 (a) y (b), observamos resultados de la muestra arcilla Estreno, donde se adjudican las mismas fases estructurales a ambas profundidades: cuarzo, montmorillonita, illita, caolinita y marialita. Sin embargo, a mayor profundidad $(0.70 \mathrm{~m})$ podríamos afirmar que la marialita casi desaparece. Mientras que en el difractograma de la Figura 4 registrada a una sola profundidad $(0.00-0.40 \mathrm{~m})$, observamos picos característicos de las siguientes fases estructurales: cuarzo, montmorillonita, seguido de la illita y caolinita. A continuación en la tabla 3 , mencionamos los códigos de cada una de las componentes de las fases obtenidas según la bases de datos en PDF del CIDD:

En todos los espectros Mössbauer registrados en las Figuras 5, 6 y 7, se obtiene los mismos resultados para las fracciones arcillosas estudiadas; observamos que todas las muestras presentan un sexteto magnético, S1 adjudicado al óxido de $\mathrm{Fe}^{3+}$ hematita y tres dobletes paramagnéticos, dos de ellos, D1 y D3 adjudicados a los sitios de $\mathrm{Fe}^{2+}$, asociados a la illita y a la montmorillonita respectivamente; y el tercer doblete, D3, adjudicado al sitio de $\mathrm{Fe}^{3+}$ en la caolinita. En la figura 5, observamos los espectros de la muestra arcilla Estrella a dos profundidades. El porcentaje de absorción del hierro para los minerales illita (D1, sitio $\mathrm{Fe}^{2+}$ ) y caolinita (D2, sitio $\mathrm{Fe}^{3+}$ ) a ambas profundidades no se altera; sin embargo, para el mineral montmorillonita (D3, sitio $\mathrm{Fe}^{2+}$ ) a mayor profundidad, se observa que existe un porcentaje mayor de absorción del hierro de este mineral. En la figura 6, observamos los espectros de arcilla Estreno a ambas profundidades, sus minerales arcillosos no son alterados por el nivel de profundidad. Y en la figura 7, podemos observar el espectro Mössbauer de la muestra arcilla Mochica donde el mineral illita (D1, sitio $\mathrm{Fe}^{2+}$ ), se puede observar a superficie, sin embargo, a mayor profundidad $(0.40-0.80 \mathrm{~cm})$, es casi escasa la absorción de este mineral. Asimismo para el mineral montmorillonita (D3, sitio $\mathrm{Fe}^{2+}$ ), el \% de absorción es similar para ambas profundidades. Caolinita (D2, sitio $\mathrm{Fe}^{3+}$ ), es el mineral donde el porcentaje de su absorción es mayor a menores profundidades.

De los resultados de ambas técnicas físicas aplicadas a estas muestras, observamos que por DRx no se observa el óxido de $\mathrm{Fe}^{3+}$, adjudicado a la hematita, dado que el porcentaje del Fe presente, en estado de valencia de $\mathrm{Fe}^{3+}$, se halla en un valor menor al $5 \%$. Sin embargo, por EMT se puede observar, y esto permite identificar que en las plantaciones de caña de azúcar existe presencia de hematita. La concentración de hierro en todas las muestras se puede determinar con la fórmula dada en la ecuación 1: [Cerón, 2001].

$$
\begin{gathered}
\text { Concentracion }(\%)=0.149\left(\frac{\mathrm{mg}}{\mathrm{cm}}\right) \frac{A_{\text {espectral }}\left(\frac{\mathrm{mm}}{\mathrm{s}}\right)}{0.01\left(\frac{\mathrm{mm}}{\mathrm{s}}\right)} \times \\
\frac{A_{\text {portamuestra }\left(\mathrm{cm}^{2}\right)}}{\text { masadelamuestra }(\mathrm{mg})} \times \frac{100}{f_{A} G} \cdots
\end{gathered}
$$

Donde $\mathrm{G}$ es la fracción de radiación $\gamma 14.4 \mathrm{keV}$ que procesa el detector con muestra, $f_{A}$, Factor de LambMössbauer. En la Tabla 3 se presentan los parámetros hiperfinos de todas las fracciones arcillosas de las muestras estudiadas, donde $\delta(\mathrm{mm} / \mathrm{s})$ representa el corrimiento isomérico, $2 \varepsilon$ el corrimiento cuadripolar, $\Delta E_{Q}$ es el desdoblamiento cuadripolar y $B_{h f}$ el campo magnético hiperfino.

Existen estudios registrados sobre la hematita bulk ó a granel y los polvos nanoestructurados de hematita $\left(\alpha-\mathrm{Fe}_{2} \mathrm{O}_{3}\right)$ en función del tamaño de la partícula, siendo su campo magnético hiperfino, $B_{h f}=52.9 \pm 0.3 \mathrm{~T}$. Esto correspondería bien al valor bulk, si se considera que la temperatura de Morin está ausente en las nanopartículas, y que el campo magnético hiperfino se reduce en aproximadamente $0,8 \mathrm{~T}$ en comparación con el valor bulk [Bødker y M $\varnothing$ rup, 2000] y [Florez et al., 2006]; por tanto, comparándolo con el valor del campo magnético de las muestras de fracción arcillosa del presente trabajo, 
se observó que son aproximadamente similares, por ello, cabe mencionar que las muestras estudiadas, son muestras de fracciones arcillosas naturales, las cuales no han seguido ningún tratamiento químico, ya sea para eliminar el óxido de hierro, como la hematita ó algunas otras fases asociadas a la arcilla, así mismo, sabemos sobre el uso del término "arcilla" y "mineral de arcilla" [Murad, 1998] y [Guggenheim y Martin, 1995]. El término "arcilla" se refiere a un material natural compuesto principalmente de minerales de grano fino, con un contenido de agua apropiado y se endurecerá cuando se seca. Aunque la arcilla generalmente contiene filosilicatos, puede contener otros materiales que imparten estas propiedades. Las fases asociadas en la arcilla pueden incluir materiales que no imparten plasticidad ni materia orgánica.
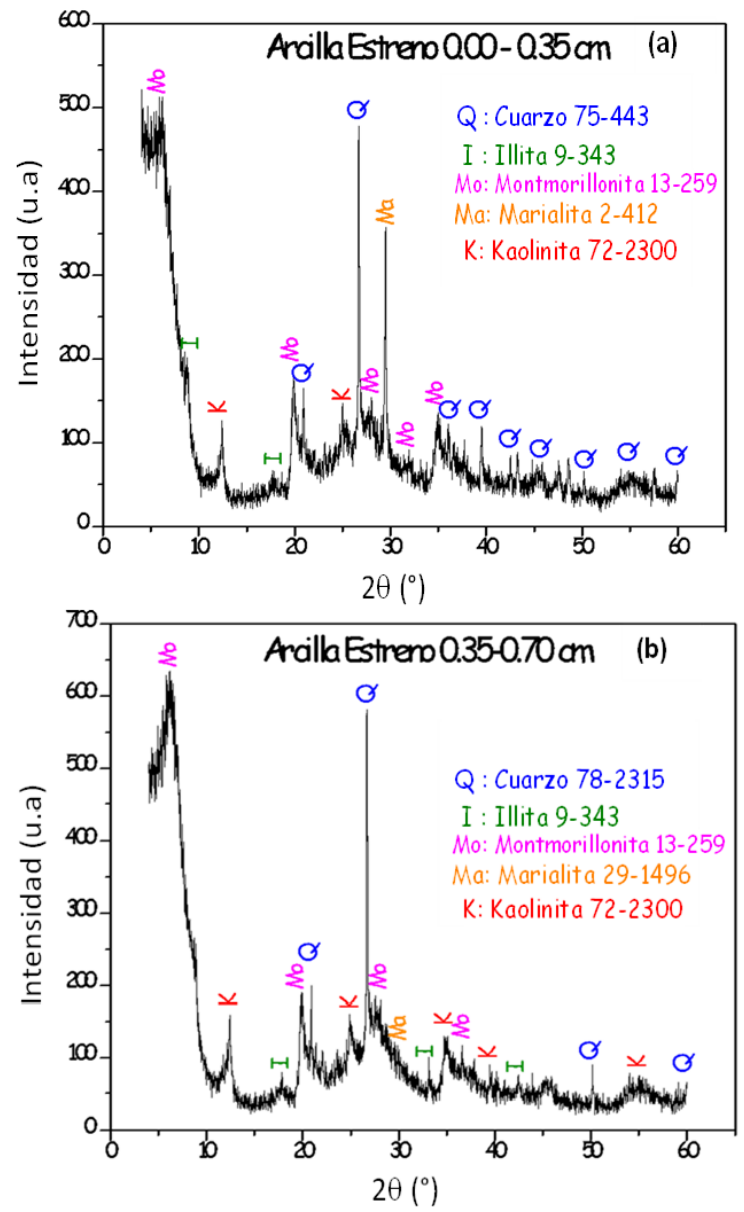

Figura 3: Patrones de difracción de rayos $\mathrm{X}$ de la muestra arcilla Estrella a una profundidad de: (a) $0.00-0.35$ m., y (b) $0.35-0.70 \mathrm{~m}$., mostrando reflexiones a ambas profundidades de las fases del cuarzo (Q), montmorillonita (Mo), illita (I), marialita $(\mathrm{Ma})$ y caolinita $(\mathrm{K})$.

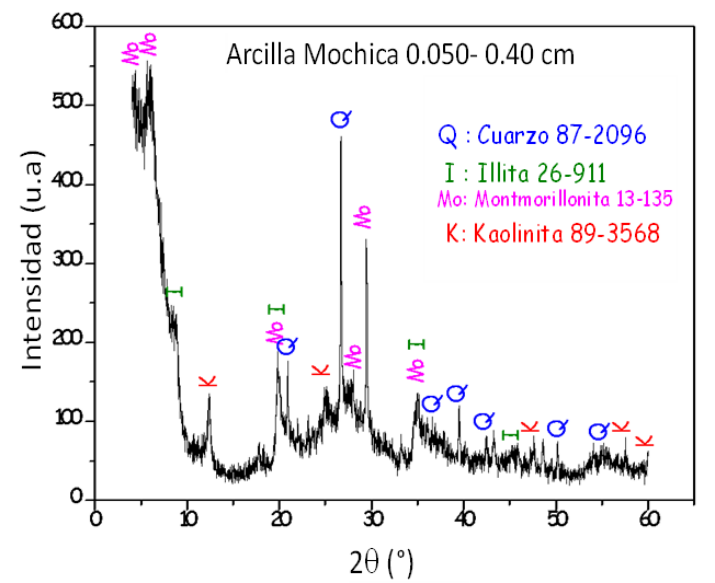

Figura 4: Patrón de difracción de rayos $\mathrm{X}$ de la muestra arcilla Mochica a una profundidad de: (a) $0.00-0.40 \mathrm{~m}$., mostrando reflexiones de las fases del cuarzo (Q), montmorillonita (Mo), caolinita (K) e illita (I).
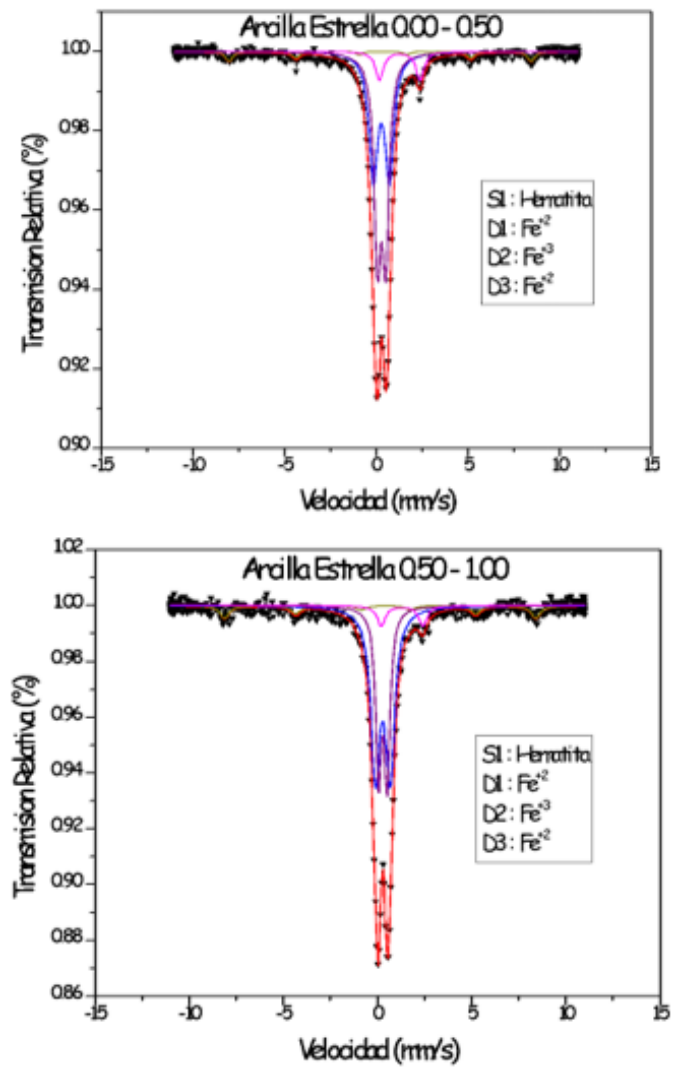

Figura 5: Espectros Mössbauer de transmisión de la muestra arcilla Estrella a diferentes profundidades: (a) $0.00-0.50$ m y (b) $0.50-1.00 \mathrm{~m}$, mostrando fases mineralógicas de sitios de óxido de hierro, S1, asignado a la hematita; y dobletes D1 y D3 asignados a la illita y montmorillonita respectivamente, y D2 asignado a la caolinita. 
El término "mineral de arcilla" se refiere a los minerales filosilicatos y minerales que imparten plasticidad a la arcilla y que se endurecen al secarse. https://www.researchgate.net/publication/ 279623865_Definition_of_clay_and_clay_mineral_ Joint_report_of_the_AIPEA_nomenclature_and_CMS_ nomenclature_committees.
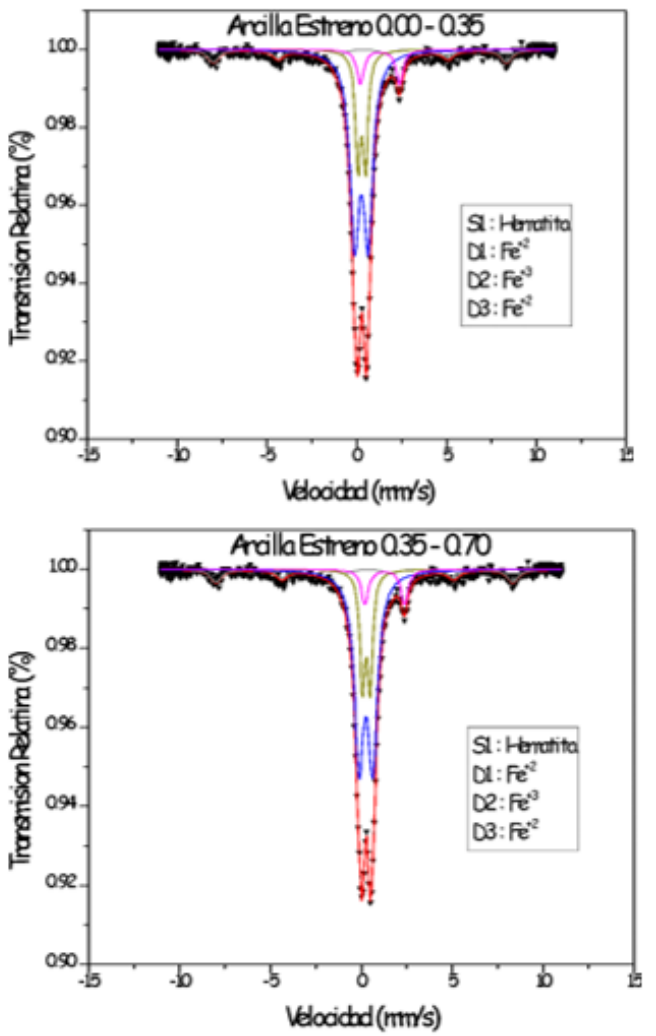

Figura 6: Espectro Mössbauer de Transmisión de la muestra Arcilla Estreno a diferentes profundidades: (a) $0.00-0.35 \mathrm{~m}$ y (b) $0.35-0.70 \mathrm{~m}$, mostrando fases mineralógicas de sitios de óxido de hierro, S1, hematita; y dobletes D1 y D3 asignados a la illita y montmorillonita respectivamente y D2 asignado a la caolinita.

Los resultados de los análisis físico-químicos nos muestran que los suelos del cultivo de caña de azúcar son fuertemente alcalinos con $\mathrm{pH}$ en el rango de 8.5-9.5 y en los análisis texturales observamos que los porcentajes de arcilla muestran ser no mayor a $45 \%$, con una clase textural de franco arenosa y franco arcilloso arenosa, lo cual nos permite obtener la presencia de arcillas a pocas profundidades. Los resultados de CE, en todas las muestras, indican ser suelos salinos a extremadamente salinos. La muestra arcilla Estrella, es la muestra que presenta valores altos de $\mathrm{CE}$ a ambas profundidades, que corresponden a son suelos extremadamente salinos. Los resultados por DRx, de esta misma muestra, presenta 5 fases estructurales, a diferencia de las otras 4 restantes; 2 de las 5 fases estructurales son composiciones que no corresponden a minerales arcillosos, ni a óxidos de hierro; éstas son marialita $\left(3 \mathrm{NaAlSi}_{3} \mathrm{O}_{8} \mathrm{NaCl}\right)$ y calcita magnesiana, que son minerales carbonatos, razón por la cual tampoco se observan por EMT, dado que no contienen Fe en su composición.
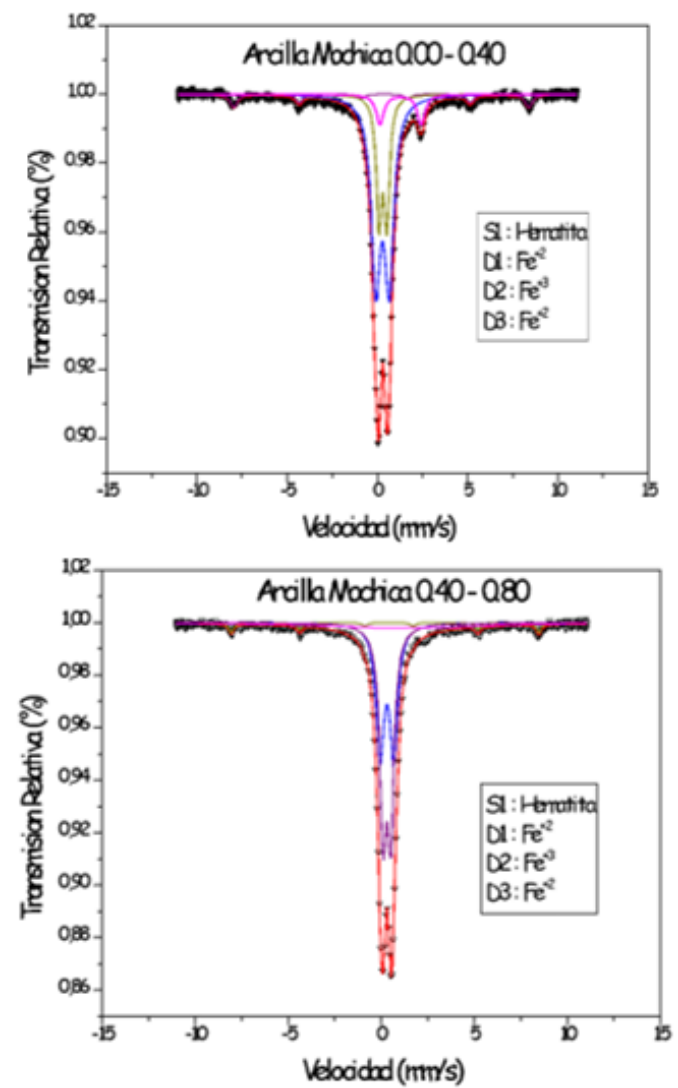

Figura 7: Espectro Mössbauer de Transmisión de la muestra Arcilla Mochica diferentes profundidades: (a) $0.00-0.40 \mathrm{~m} \mathrm{y}$ (b) $0.40-0.80 \mathrm{~m}$, mostrando fases mineralógicas de sitios de óxido de hierro, S1, asignado a la hematita; y dobletes D1 y D3 asignados a la illita y montmorillonita respectivamente y D2 asignado a la caolinita.

\section{Conclusiones}

Identificamos en las fracciones arcillosas de los suelos de las plantaciones de caña de azúcar, la presencia de sextetos magnéticos, adjudicado al óxido de $\mathrm{Fe}^{3+}$ : hematita; sitios con cationes de $\mathrm{Fe}^{2+}$ y $\mathrm{Fe}^{3+}$, localizados en las arcillas de illita, montmorillonita y caolinita. Así mismo otros minerales como cuarzo, marialita y calcita magnesiana. Los resultados físico-químicos, nos permiten determinar que los suelos se encuentran en un rango 


\begin{tabular}{|c|c|c|c|c|}
\hline MUESTRAS & Minerales/sitios Fe & $\begin{array}{c}\delta \\
(\mathrm{mm} / \mathrm{s})\end{array}$ & $\begin{array}{c}2 \varepsilon / Q U A \\
(\mathrm{~mm} / \mathrm{s})\end{array}$ & $\begin{array}{l}B_{h f} \\
(\mathrm{~T})\end{array}$ \\
\hline \multirow{4}{*}{$\begin{array}{l}\text { Arcilla } \\
\text { Estrella } \\
0.00-0.50 \mathrm{~m}\end{array}$} & S1, Hematita & 0.294 & -0.18 & 51.04 \\
\hline & D1, Montmorillonita $\left(F e^{2+}\right)$ & 0.277 & 0.863 & \\
\hline & D2, Illita $\left(F e^{2+}\right)$ & 1.285 & 2.213 & \\
\hline & D3, Caolinita $\left(F e^{3+}\right)$ & 0.287 & 0.451 & \\
\hline \multirow{4}{*}{$\begin{array}{l}\text { Arcilla } \\
\text { Estrella } \\
0.50-1.00 \mathrm{~m}\end{array}$} & S1, Hematita & 0.30 & -0.30 & 51.19 \\
\hline & D1, Montmorillonita $\left(F e^{2+}\right)$ & 0.286 & 0.784 & \\
\hline & D2, Illita $\left(F e^{2+}\right)$ & 1.308 & 2.207 & \\
\hline & D3, Caolinita $\left(F e^{3+}\right)$ & 0.292 & 0.461 & \\
\hline \multirow{4}{*}{$\begin{array}{l}\text { Arcilla } \\
\text { Estreno } \\
0.00-0.35 \mathrm{~m}\end{array}$} & S1, Hematita & 0.272 & -0.18 & 50.58 \\
\hline & D1, Montmorillonita $\left(F e^{2+}\right)$ & 0.261 & 0.765 & \\
\hline & D2, Illita $\left(F e^{2+}\right)$ & 1.296 & 2.181 & \\
\hline & D3, Caolinita $\left(F e^{3+}\right)$ & 0.282 & 0.425 & \\
\hline \multirow{4}{*}{$\begin{array}{l}\text { Arcilla } \\
\text { Estreno } \\
0.35-0.70 \mathrm{~m}\end{array}$} & S1, Hematita & 0.277 & -0.181 & 50.88 \\
\hline & D1, Montmorillonita $\left(F e^{2+}\right)$ & 0.275 & 0.748 & \\
\hline & D2, Illita $\left(F e^{2+}\right)$ & 1.249 & 2.260 & \\
\hline & D3, Caolinita $\left(F e^{3+}\right)$ & 0.292 & 0.426 & \\
\hline \multirow{4}{*}{$\begin{array}{l}\text { Arcilla } \\
\text { Mochica } \\
0.00-0.40 \mathrm{~m}\end{array}$} & S1, Hematita & 0.299 & -0.196 & 50.69 \\
\hline & D1, Montmorillonita $\left(F e^{2+}\right)$ & 0.276 & 0.747 & \\
\hline & D2, Illita $\left(F e^{2+}\right)$ & 1.280 & 2.278 & \\
\hline & D3, Caolinita $\left(F e^{3+}\right)$ & 0.297 & 0.438 & \\
\hline \multirow{4}{*}{$\begin{array}{l}\text { Arcilla } \\
\text { Mochica } \\
0.40-0.80 \mathrm{~m}\end{array}$} & S1, Hematita & 0.299 & -0.206 & 51.26 \\
\hline & D1, Montmorillonita $\left(F e^{2+}\right)$ & 0.289 & 0.804 & \\
\hline & D2, Illita $\left(F e^{2+}\right)$ & 1.478 & 2.131 & \\
\hline & D3, Caolinita $\left(F e^{3+}\right)$ & 0.297 & 0.438 & \\
\hline
\end{tabular}

Tabla 4: Parámetros Mössbauer de las muestras del Distrito de Pomalca. 
entre suelos salinos a extremadamente salinos. Éstos resultados estarán a disposición de los profesionales de la Empresa Pomalca S.A.A. Continuando en nuestra línea de investigación, para futuros trabajos incluiremos métodos que nos permitan la estimación cuantitativa de las fases cristalinas.

\section{Agradecimientos}

Las (os) autores (as) agradecen la colaboración a los miembros de los Laboratorios de Análisis de Suelos, de Arqueometría y de Difractometría de Rayos X de la FCF; UNMSM por permitirnos el uso de sus instrumentos, materiales y equipos para la investigación de estas muestras.

\section{Referencias}

[Ace04] Acevedo, O.†, Ortiz E., Cruz M. y Cruz E. (2004). El papel de óxidos de hierro en suelos. Redalyc. (22), 4, 485-497.

[Bas20] Báscones, A. (2020). Caracterización de minerales de arcilla y óxidos de hierro mediante espectroscopía de reflectancia difusa (VNIR-SWIR). Revista de Teledetección, (55). 49-57.

[Bød00] Bødker, F. y Mørup, S. (2000). Size dependence of the properties of hematite nanoparticles. Europhys. Lett. 52 (2). 217-223.

[Brav00] Bravo, J., Fabián, J.; Cerón, M., Trujillo, A., Huaypar, Y. y Mejia, M. (2000). Manual de Laboratorio de Análisis de Suelos. Laboratorio de Análisis de Suelos. Facultad de Ciencias Físicas, Universidad Nacional Mayor de San Marcos. Lima, Perú.

[Bra95] Brand, R. (1995). NORMOS Mössbauer Fitting Program. User's Guide. Wissenschaftlich Elektronik $\mathrm{GmbH}$, Starnberg.

[Bur04] Burt, R. (Ed.). (2004). Soil Survey Laboratory Methods Manual. Soil Survey Investigations Report No. 42. Version 4.0. Natural Resources Conservation Service. United States Department of Agriculture. 700 ps.

[Hum12] Bustos, H., Lozano, D.. Martínez, Y., Pinilla, M. y Alcázar, G. (2012). Characterization of mineral phases of agricultural soil samples of Colombian coffee using Mössbauer spectroscopy and X-ray diffraction. Hyperfine Interact 208: 13-18.

[Cer01] Cerón, M. (2001). Estudio mineralógico de suelos agrícolas por espectroscopía Mössbauer. Universidad Nacional Mayor de San Marcos; UNMSM. Perú: Lima..

[Cer11] Cerón, M., Bravo, J., y Mejía, M. (2011). Mineralogy of the clay fraction of soils from the Moray Cusco archaeological site: a study by energy dispersive X-ray fluorescence, X-ray diffractometry and Mössbauer spectroscopy. Hyperfine Interactions. 203, (1-3), 133-141.

[Cer17] Cerón, M., Bravo, J. y Reyes, F. (2017). A Preliminary Study on Organoclays from Two Peruvian Clay Pits. Malaysian Journal of Soil Science. 21, 6371.

[Cor03] Cornell, R. y Schwertmann, U. (2003). The iron oxides: Structure, Properties, Reactions, Ocurrences and Uses. 2nd Edition Wiley-VCH.Gmbh and Co. KgAa. Federal Republic of Germany.

[Dan02] Dane, J. and Topp, G. (Eds.). (2002). Methods of Soil Analysis. Part 4. Physical Methods. Soil Sci. Soc. Am. Book Series No. 5. Soil Science Society of America, Inc. Madison, Wis. 1692 ps.

[Flo] Flores, L. y Alcala, J. (2010). Manual de Procedimientos Analíticos. Laboratorio de Física de Suelos. Instituto de Geología. Universidad Nacional Autónoma de México. https : //www.geologia. unam.mx/igl/deptos/ edafo/lfs/MANUAL $\% 20 D E L \% 20 L A B O R A T O R I 0 \% 20 D E \%$ 2OFISICA $\% 20 D E \% 20$ SUELOS 1 .pdf

[Flo06] Florez, J., Mazo-Zuluaga, J., Casanova, H. y Restrepo, J. (2006). Estudio Mössbauer de hematita molida mecánicamente. Revista Colombiana de Física. 38 (4).

[Gug95] Guggenheim, S. y Martin, R. (1995). Definition report of clay and clay mineral: Joint the AIPEA and CMS Nomenclature Committees. Clay Minerals (30) 257-259.

[Hid10] Hidalgo, C., Etchevers, J., Martínez-Richa, A., Yee-Madeira, H., Calderon, H., Vera-Graziano, R., Matus, F. (2010). Mineralogical characterization of the fine fraction $(<2 \mu \mathrm{m})$ of degraded volcanic soils and tepetates in Mexico. Appl. Clay Sci. 49, 348-358.

[Klu86] Klute A. (Ed.). (1986). Methods of Soil Analysis. Part I. Physical Methods. 2nd ed. Agron. Monogr. 9. Soil Science Society of America, Inc., Madison, Wis. 
[Man11] Manzo, V., Pizarro, C., Rubio, M., Cavalcante, L., Garrg, V. y Fabris, J. (2011). Preparative treatment with $\mathrm{NaOH}$ to selectively concentrate iron oxides of a Chilean volcanic soil material to produce effective heterogeneous Fenton catalyst. Hyperfine Interactions. (203).59-66.

[Mej13] Mejía, M. (2013). Caracterización mineralógica de los suelos tropicales de la reserva forestal de la Universidad Agraria de la Selva por difractometría de rayos X y espectroscopía Mössbauer. Universidad Nacional Mayor de San Marcos; UNMSM. Perú: Lima.

[Mij97] Mijovilovich, A. (1997). Estudio Mössbauer de óxidos e hidróxidos de Fe: Aplicación al estudio de suelos. (Tesis doctoral). UNBA, Argentina.

[Mij99] Mijovilovich, A., Morras, H., Causevic, H., Saragovi, C. (1999). Mössbauer study of the mineralogy in two different Argentine soils. Hyperfine Interact. 122, 83-95.

[Min03] Ministerio de Agricultura. (2003). Programa para el Desarrollo de la Amazonía. Caracterización de las zonas cafetaleras en el Perú. Informe final, Lima, Perú.

[Moo97] Moore, D. and Reynolds, R. (1997). X-Ray Diffraction and the Identification and Analysis of Clay Materials, 2da. Edition, Oxford University Press.

[Mur98] Murad, E. (1998). Clays and clay minerals: What can Mossbauer spectroscopy do to help understand them?. Hyperfine Interactions (117). 39-70.

[Mur07] Murray, H.H. (2007). Applied Clay Mineralogy: Occurrences, Processing and Application of Kaolins, Bentonites, Palygorskite-Sepiolite, and Common Clays. Elsevier, Amsterdam.

[Nim08] Nima, F. (2008). Caracterización mineralógica de la fracción arcillosa de vertisols de la parcela U.N.P - San Lorenzo, Piura. Universidad Nacional de Piura. Perú: Piura.
[Nig04] Nigam, V., Setua, D., Mathur, G. and Kar, K. (2004). Epoxy-montmorillonite clay nanocomposites: Synthesis and characterization. Journal of applied polymer science. 93(5): 2201-2210.

[Pat14] Patel, H. (2014). Nanoclays: Synthesis, Characterization and Applications. Inglaterra. Scholars World.

[Per06] Perugachi, B., Paredes, V. y Cornejo, M.(2006). Las Nanoarcillas y sus potenciales aplicaciones en el Ecuador. Revista Tecnológica ESPOL. 19 (1). 121124.

[Piz17] Pizarro, C., Escudey, M., Gacitua, M., Domingos, J. (2017). Iron-bearing minerals from soils developing on volcanic materials from southern Chile. Mineralogical characterization supported by Mössbauer spectroscopy. Journal of Soil Science and Plant Nutrition. 17 (2), 341-365.

[Ric85] Richards, L. (1985). Diagnóstico y Rehabilitación de Suelos Salinos y Sódicos. 6ta. Ed. Editorial Limusa. México, D. F. 172 p. (USDA, Manual 60).

[Suf19] Sufardi, Arabia, Khairullah, Karnilawati y Nurnikmat. (2019). Distribution of $\mathrm{Al}, \mathrm{Fe}$, and $\mathrm{Si}$ oxides in three soil orders in dryland of Aceh Besar, Indonesia. IOP Conf. Series: Earth and Environmental Science,,(393). 012081. Recuperado de https://iopscience.iop.org/article/10. 1088/1755-1315/393/1/012081/pdf.

[Ste98] Stevens, J. (Editor). (1998). Mössbauer Minerals Handbook, Mössbauer Effect Data Center, Ashville, NC.

[Tan93] Tanejaa, S. y Rajb, D. (1993). Mössbauer and X-ray studies of soils. Nucl. Instrum. Methods Phys. Res., B Beam Interact. Mater. Atoms 76(1-4), 233-235.

[Tho02] Thompson, L. y Troeh, R. (2002). Los suelos y su fertilidad. Reverté S.A. España: Barcelona.

[Tru02] Trujillo, A. (2002). Caracterización mineralógica de suelos agrícolas por difracción de rayos $\mathrm{X}, \mathrm{y}$ espectroscopia Mössbauer. Universidad Nacional de Piura, Perú: Piura. 